\title{
Impact of Designed Nursing Educational Protocol on Health Promotion for Patients Undergoing Coronary Artery Stent Outcome
}

\author{
Ghada Th. Mohammed, Zienab A. Mohammed, Hossam H. Al-Araby. \\ Nursing specialist at cardiac catheterization unit, Master Degree in Adult Nursing, Assiut University, Egypt. \\ Professor of Medical Surgical Nursing, Faculty of Nursing, Assiut University, Egypt. \\ Professor of Cardiology, Faculty of Medicine, Assiut University, Egypt.
}

\begin{abstract}
Aim: determine the educational needs of patients with coronary artery stent, design a nursing educational protocol, and evaluate the impact of implementing the nursing educational protocol on patient's knowledge, and prevention /reduction of complications. Methods: the study was conducted in catheterization Lab at Assiut University Hospital. A convenience sample all adult and mentally competent male and female (60) patients. Tools utilized to collect data were Patient assessment, Pre-Post test questionnaire \& Patients Complications assessment. Results: As regard to the mean knowledge scores of post educational protocol: There is a significant improvement of all parameters in response to the given information among the study sample with a percentage (100\%) in comparison with the control sample that still have poor knowledge score. As regard to Health promotion of participants: there is no significant statistical differences between control and study samples in relation to local and circulatory complications immediate post and after 3 months. Conclusion: Application of teaching protocol when dealing with coronary artery stent patients shows a significant improvement in Patient's knowledge. Recommendations: Equip the cardiac catheterization unit with simply illustrated guidelines protocol covering PCI Procedure and coronary stent knowledge pre/post cardiac catheterization.
\end{abstract}

\section{Keywords: Nursing Educational Protocol \& Health Promotion, Coronary Artery Stent.}

\section{Introduction}

Percutaneous coronary intervention (PCI) is one of the two coronary revascularization techniques currently used in the treatment of ischemic heart disease, the other being coronary artery bypass grafting $(\mathrm{CABG})$. A minimally invasive device for opening blocked coronary arteries, known as the coronary stent, has been developed more recently to avoid many of the complications of bypass surgery. A typical stent is formed as a mesh tube, constructed from metal wire commonly made of stainless steel or other metals. (Sujata, 2010).Percutaneous transluminal coronary angioplasty (PTCA), formerly known as balloon angioplasty. (European Society of Cardiology, 2010)

PCI is performed to open blocked coronary arteries caused by coronary artery disease (CAD) and to restore arterial blood flow to the heart tissue without open-heart surgery. (Roger et al., 2010).

The PCI is often combined with the permanent placement of a small wire mesh tube called a stent to help prop the artery open and decrease the chance of narrowing it again. Some stents are coated with medication to help keep the artery open (drug-eluting stents) while others are not (bare-metal stents). (Lisa, et al., (2010).

Unlike CABG, stenting is considered minimally invasive because it involves no major incisions. Performed with local anesthesia and mild sedation, the procedure usually takes about an hour, but it can take longer if multiple stents are required. Patients who undergo stenting experience significantly less discomfort and a shorter recovery time than those who undergo CABG (Morton \& Fontaine, 2009).

Stenting isn't risk-free. Sometimes, a blood clot can form in the stent and cause a sudden narrowing or even a complete blockage. To prevent this process, which is known as in-stent thrombosis, patients who undergo stenting must take one or more bloodthinning drugs after the procedure. These include aspirin, which usually must be taken indefinitely, and clopidogrel, which is usually prescribed for at least one and up to 12 months (Lewis et al., 2007).

Types of stents: First-generation stent were made of bare metal. Although bare-metal stent almost eliminated the risk of the artery collapsing, they only modestly reduced the risk of restenosis. About 25\% of all coronary arteries treated with bare-metal stents would close up again, usually within about six months. So doctors and companies began testing stents which were coated with drugs that interrupted the process of restenosis. These are called drugeluting stents (George \& Josh, 2010).

Stenting complications include mild complications as minor discomfort at the site of the puncture in the groin or the arm; also patients may experience brief episodes of angina while the balloon is inflated, and 
briefly blocking the flow of blood in the coronary artery, moderate complications as thrombosis, restenosis, inflammation, hyperplasia, bleeding, drug reaction allergic reaction ,and severe complications as, embolism, perforation, $\mathrm{CABG}$ or total occlusion of the artery, stent failure, myocardial ischemia, stent migration and death. (American Heart Association, 2010).

Patients are maintained on aspirin indefinitely after PCI to prevent future thrombotic events (for example, unstable angina or heart attack). In patients who receive stents, an additional anti-platelet agent [in most instances clopidogrel] is given in conjunction with aspirin for one to three months (Osborn et al., 2010).

It is very important for the patient undergoing PCI to be informed about the instructions before, during and after the procedure to avoid complications, Nursing care for PTCA and stent insertion includes: Immediately after the procedure, the patient is at risk of arrhythmia and myocardial infarction. The patient may be required to stay in bed for several hours. When the patient returns to the ward or department following the procedure, cardiac monitoring is recommended, Observations of blood pressure (BP), heart rate, respirations, and temperature should be recorded. The limb that has been used for the procedure needs to be closely monitored for signs of poor arterial circulation. (British Heart Foundation, 2008).

\section{Significance of the Study}

In catheterization lab of Assiut University Hospital, around 90 patients with coronary artery stent has been operated monthly without an organized educational protocol for the patients either before or after the procedure.

Therefore, this study was the first in this geographical location which helped such group of patients to maintain proper functioning of the coronary stent, identify and report symptoms indicative coronary artery stent, complications as well to maintain his / her wellness. Furthermore, results of this study could be helpful for health professionals especially nurses in planning and implementing care for such group of patients in the future.

\section{The aims of the study}

\section{General aim}

To evaluate the impact of designed nursing educational protocol on health promotion for patients undergoing coronary artery stent outcome.

\section{Specific aims}

- Determine the educational needs of patients with coronary artery stent.
- Develop a nursing educational protocol based on predetermining the educational needs.

- Evaluate the impact of implementing the nursing educational protocol on patient's knowledge and on prevention /reduction of complications.

\section{Research hypotheses}

To fulfill the aim of the study, for study group patients the following hypotheses are formulated:

- The mean knowledge scores exposed to the educational protocol was higher than that of their pre-protocol mean scores.

- The frequency of coronary artery stent complications was lesser among study group patients compared to those among the control group ones.

\section{Operational definitions}

Health promotion

The prevention or/reduction of complications.

Coronary artery stent

A small tube-like device made of stainless steel or other material used to hold open a blocked artery.

\section{Patients \& Method}

\section{Research design}

The quasi-experimental research design was utilized in this study.

\section{Study variables}

The independent variable in this study is the nursing educational protocol while the dependent variables are: patient's knowledge, skills and on prevention /reduction of complications. The present study was portrayed under topics as the following: technical design, operational design, administrative design, and statistical design.

\section{Subjects}

A convenience sample of sixty adult male and female patients aged from (18-65 years old) who are scheduled for non-emergency coronary artery stent. They were assigned to both study and control samples.

Patients in the study sample (30 patients) were received nursing educational protocol while the control sample (30 patients) were received routine care.

Patients were selected according to the following criteria:

- Normal prothrombin time.(10-14 seconds) (Urden, Stacy, \& cough, 2006)

- Normal renal function test.( BUN is 5 to $25 \mathrm{mg} / \mathrm{dl}$, creatinine is 0.5 to $1.5 \mathrm{mg} / \mathrm{dl}$ ) (Urden, Stacy, \& cough, 2006)

- Hemodynamically stable. (e.g. within normal vital signs, chest free, controlled blood sugar ...etc ) 


\section{Setting}

The study was conducted in a Coronary Catheterization lab of Assiut University Hospital.

Tools

\section{I: Patient with coronary artery stent assessment} tool

Covers the following parts

Part (1): Demographic characteristics of both study and control sample (e.g., age, sex, marital status, the level of education, and occupation. It included 5 Questions from 1 to 5.

Part (2): a physical assessment. Included medical history (Presenting Symptoms, Risk factors, family history, medical \& surgical history), cardiac physical examination (physical problems), and psychological problems include fear and anxiety.

\section{II: Pre/ Post-test questionnaire tool}

Used to assess patient's Knowledge about coronary artery stents :( its function, when, how to apply, types, preparations before the procedure, medications before \& after the procedure, complications and signs of risk in which the patient must call the doctor.).

\section{Scoring system}

Each right answer was given two degrees. The total scores were 70 . Those who obtained less than $50 \%$ were considered having a poor level. While those who obtained $50 \%$ were considered having a fair level and more than $50 \%$ were considered having a good level of knowledge.

\section{III: Coronary artery stent patient's complications} assessment

It was used to assess expected complications that might develop among patients with coronary artery stent after implementation of the nursing educational protocol. It included signs and symptoms of complications related to coronary stents, it consisted the following:

Local complications defined in this study as the presence of infection at the catheter insertion site and Allergic reaction to the dye.

Circulatory complications: included in this study as presence of

- Bleeding at the catheter insertion site.

- Hematoma: a Blood clot or damage to the blood vessel at the insertion site.

- In-stent Thromboses: Blood clot within the vessel treated by PCI or stent.

- Cardiac dysrhythmias or arrhythmias (abnormal heart rhythms).

- Myocardial infarction duo to restenosis.

- Chest pain or discomfort / readmitted to CCU.

- Perforation: Rupture of the coronary artery, requiring open-heart surgery

Urinary complications included in this study as presence of
- Urinary retention.

- Urinary tract infection.

- Kidney failure

Gastrointestinal complications included in this study as presence of

- Nausea and/or vomiting.

- Constipation

Late complications included in this study as presence of:

- Fever and/or chills.

- Increased pain, redness, swelling, or bleeding or other drainage from the insertion site.

- Coolness, numbness, and/or tingling, or other changes in the affected extremity.

- Chest pain/pressure, profuse sweating, dizziness, and/or fainting.

- Radial artery occlusion

Designed nursing educational protocol

It was developed by the researcher from literature reviews, researcher experience and opinions of the medical and nursing expertise based on patient's assessment needs to maintain health promotion for patients. The teaching booklet was revised and modified based on the expertise comments, it was written in Arabic using simple language with illustrations and it was concerning knowledge about:

- A brief illustration of the anatomy and physiology of the heart and coronary arteries.

- Information about coronary artery disease (definition, causes, symptoms, and nursing care)

- Information about coronary artery stents (what's it, function, indications, types, it's complications... etc.)

- Information about medical therapy before and after coronary artery stents, lifestyle modifications, the importance of follow up and when it is necessary to seek medical help and immediately go to the hospital.

Tools testing and pilot study

The final form of the proposed protocol and assessment tools was checked by a panel of experts for content validity and reliability.

To assess clarity and feasibility of contents

- A pilot study was implemented on (10\%) 6 patients to examine the feasibility of the study. Analyzes of the pilot study revealed that minimal modifications are required. These modifications were done and the subjects were done excluded from the actual study. The purpose of the pilot study was:

- To ascertain the relevance of the tools.

- To detect any problem peculiar to the statements clarity that might interfere with the process of data collection.

- To estimate the time needed to complete the interview schedule. 


\section{Method}

This study was carried out in 3 phases

- The first phase is the preparatory phase in which the researcher was designed and tested the proposed teaching protocol and assessed patients knowledge in this regard (using tool 1).

- The second phase is the implementation phase it was directed toward patients based on a finding of the exploratory phase, the nursing educational protocol was developed, after extensive literature review considering patients needs and their levels of understanding.

Each patient obtained a copy of the nursing educational protocol booklet that included all the training contents.

- The last phase is the evaluation phase, in which the patient's knowledge was evaluated immediately post protocol implementation using (tool 2,3) and after 3 months using (tool 3).

Careful teaching and reinforcement of teaching was performed according to the patient nursing needs to ensure their understanding. The whole period for teaching protocol implementation was 1 year.

\section{Administrative design}

Permission to carry out the study was obtained from the responsible hospital authorities of the catheterization lab at Assiut University Hospital, After explaining the aim of the study and the nursing educational protocol to obtain their cooperation also oral and written permission from the participating patients in catheterization lab was obtained.

\section{Ethical considerations}

- The research proposal was approved by Ethical Committee in the faculty of nursing.

- Written consent was obtained from patient's that are participated in the study, after explaining the nature and purpose the study.

- Confidentiality and anonymity were assured.

- Study subject has the right to refuse to participate and or withdraw from the study without any rational at any time.

- Study subject privacy was considered during collection of data.

\section{Statistical design}

The data obtained had reviewed, prepared for computer entry, coded, analyzed and tabulated. Descriptive statistics (frequencies and percentages, mean and standard deviation, i.e.) were done using computer program (SPSS) version(22). Independent sample T-test, Chi-square and One-way-ANOVA tests used in the relationship between study and control groups' physically \& psychologically, knowledge and complications. It's considered significant when $P$. value less than (0.05).

\section{Limitation of the study}

The result of this study could not be generalized due to the small sample size, not representative of all population, and also not geographically distributed because of the sample. 


\section{Results}

Table (1): Comparison between the study and control samples in relation to demographic data.

\begin{tabular}{|c|c|c|c|c|c|}
\hline \multirow{2}{*}{ Items } & \multicolumn{2}{|c|}{ Study sample } & \multicolumn{2}{|c|}{ Control sample } & \multirow{2}{*}{$P$ value } \\
\hline & N. $(n=30)$ & $\%$ & N. $(n=30)$ & $\%$ & \\
\hline \multicolumn{5}{|l|}{ Gender } & \multirow{3}{*}{0.095} \\
\hline Male & 23 & 76.7 & 20 & 66.7 & \\
\hline Female & 7 & 23.3 & 10 & 33.3 & \\
\hline \multicolumn{5}{|l|}{ Age } & \multirow{5}{*}{0.530} \\
\hline $18-40$ & 1 & 3.3 & 2 & 6.7 & \\
\hline $40-60$ & 20 & 66.7 & 21 & 70.0 & \\
\hline More than 60 & 9 & 30.0 & 7 & 23.3 & \\
\hline Mean \pm SD & \multicolumn{2}{|c|}{$55.9 \pm 9.1$} & \multicolumn{2}{|c|}{$53.7 \pm 9.7$} & \\
\hline \multicolumn{5}{|l|}{ Marital status } & \multirow{5}{*}{0.981} \\
\hline Single & 1 & 3.3 & 0 & 0.0 & \\
\hline Married & 28 & 93.3 & 29 & 96.7 & \\
\hline Divorced & 0 & 0.0 & 0 & 0.0 & \\
\hline Widowed & 1 & 3.3 & 1 & 3.3 & \\
\hline \multicolumn{5}{|l|}{ Level of education } & \multirow{6}{*}{0.814} \\
\hline High education & 3 & 10.0 & 0 & 0.0 & \\
\hline Secondary education & 3 & 10.0 & 6 & 20.0 & \\
\hline Basic education & 3 & 10.0 & 4 & 13.3 & \\
\hline Read and write & 4 & 13.3 & 1 & 3.3 & \\
\hline Illiterate & 17 & 56.7 & 19 & 63.3 & \\
\hline \multicolumn{5}{|l|}{ Occupation } & \multirow{4}{*}{0.323} \\
\hline Employee & 13 & & 8 & 26.7 & \\
\hline Farmer & 10 & 33.3 & 13 & 43.3 & \\
\hline House wife & 7 & 23.3 & 9 & 30.0 & \\
\hline
\end{tabular}

Independent sample T-test $*$ Statistical significant differences $(p \leq 0.05$

Table (2): Comparison between the study and control samples in relation to patient.

A- Physically data. B-Cardiac physical examination (physical problems) C- Psychological problems.

\begin{tabular}{|c|c|c|c|c|c|}
\hline \multirow{2}{*}{ Items } & \multicolumn{2}{|c|}{ Study sample } & \multicolumn{2}{|c|}{ Control sample } & \multirow{2}{*}{$P$ value } \\
\hline & N. (30) & $\%$ & N. (30) & $\%$ & \\
\hline \multicolumn{6}{|l|}{ Inspection } \\
\hline \multicolumn{5}{|l|}{ Face } & \multirow{3}{*}{0.133} \\
\hline Redness & 25 & 83.3 & 27 & 90.0 & \\
\hline Pale & 5 & 16.7 & 3 & 10.0 & \\
\hline \multicolumn{5}{|l|}{ Jugular veins } & \multirow{2}{*}{-} \\
\hline Normal & 30 & 100.0 & 30 & 100.0 & \\
\hline \multicolumn{5}{|l|}{ Thorax } & \multirow{2}{*}{-} \\
\hline Normal & 30 & 100.0 & 30 & 100.0 & \\
\hline \multicolumn{5}{|l|}{ Abdomen } & \multirow{3}{*}{$0.003 * *$} \\
\hline Normal & 30 & 100.0 & 28 & 93.3 & \\
\hline Distention & 0 & 0.0 & 2 & 6.7 & \\
\hline \multicolumn{5}{|l|}{ Nail beds } & \multirow{2}{*}{-} \\
\hline Normal & 30 & 100.0 & 30 & 100.0 & \\
\hline \multicolumn{5}{|l|}{ Lower extremities } & \multirow{4}{*}{$0.000 * * *$} \\
\hline Normal & 30 & 100.0 & 19 & 63.3 & \\
\hline Pain & 0 & 0.0 & 9 & 30.0 & \\
\hline Ulcer & 0 & 0.0 & 2 & 6.7 & \\
\hline
\end{tabular}




\begin{tabular}{|c|c|c|c|c|c|}
\hline \multirow{2}{*}{ Items } & \multicolumn{2}{|c|}{ Study sample } & \multicolumn{2}{|c|}{ Control sample } & \multirow{2}{*}{$P$ value } \\
\hline & N. (30) & $\%$ & N. (30) & $\%$ & \\
\hline \multicolumn{6}{|l|}{ Palpation } \\
\hline \multicolumn{5}{|l|}{ Pulse } & \multirow{3}{*}{$0.043^{*}$} \\
\hline Normal & 30.0 & 100.0 & 29 & 96.7 & \\
\hline Hyper dynamic pulse & 0 & 0.0 & 1 & 3.3 & \\
\hline \multicolumn{5}{|l|}{ * Capillary refill } & \multirow{2}{*}{-} \\
\hline Normal & 30 & 100.0 & 30 & 100.0 & \\
\hline \multicolumn{5}{|l|}{ * Edema } & \multirow{3}{*}{$0.000 * * *$} \\
\hline Not present & 30 & 100.0 & 27 & 90.0 & \\
\hline Present pitting & 0 & 0.0 & 3 & 10.0 & \\
\hline \multicolumn{6}{|l|}{ Auscultation } \\
\hline \multicolumn{5}{|l|}{ * Heart sound } & \multirow{3}{*}{$0.000 * * *$} \\
\hline Normal & 30 & 100.0 & 26 & 86.7 & \\
\hline Abnormal & 0 & 0.0 & 4 & 13.3 & \\
\hline \multicolumn{5}{|l|}{ * Chest } & \multirow{5}{*}{$0.008 * *$} \\
\hline Clinically free & 29 & 96.7 & 26 & 86.7 & \\
\hline Crepitating & 0 & 0.0 & 1 & 3.3 & \\
\hline Wheezing & 1 & 3.3 & 2 & 6.7 & \\
\hline Other & 0 & 0.0 & 1 & 3.3 & \\
\hline \multicolumn{5}{|l|}{ * Blood Pressure } & \multirow{3}{*}{$0.000 * * *$} \\
\hline Normal & 30 & 100.0 & 21 & 70.0 & \\
\hline Hypertension & 0 & 0.0 & 9 & 30.0 & \\
\hline \multicolumn{6}{|c|}{ C- Psychological problems } \\
\hline \multicolumn{5}{|c|}{ * Fearful } & \multirow{3}{*}{$0.080^{*}$} \\
\hline Yes & 9 & 30.0 & 24 & 80.0 & \\
\hline No & 21 & 71.0 & 6 & 20.0 & \\
\hline \multicolumn{5}{|l|}{ *Anxious } & \multirow{3}{*}{$0.003^{* *}$} \\
\hline Yes & 30 & 100.0 & 28 & 93.3 & \\
\hline No & 0 & 0.0 & 2 & 6.7 & \\
\hline
\end{tabular}

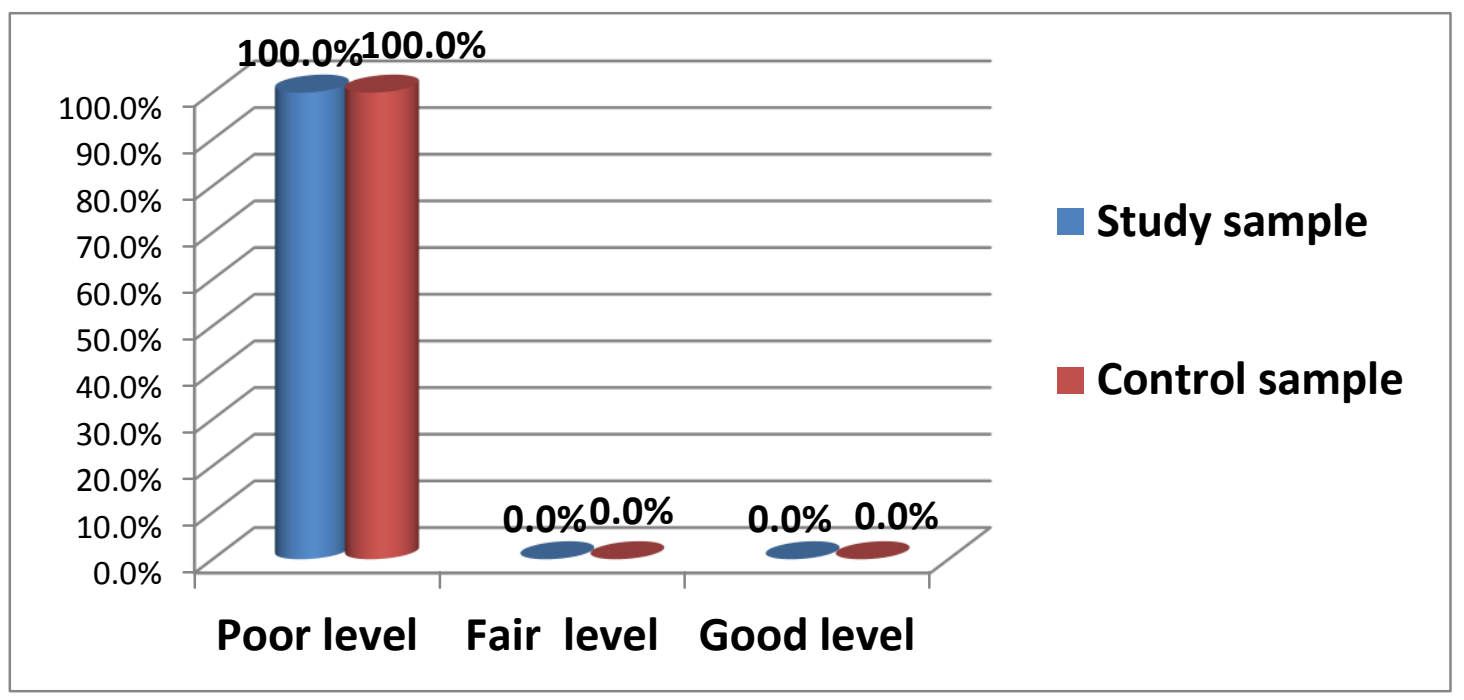

Fig. (1): Patients' knowledge pre- test about coronary artery stent. 


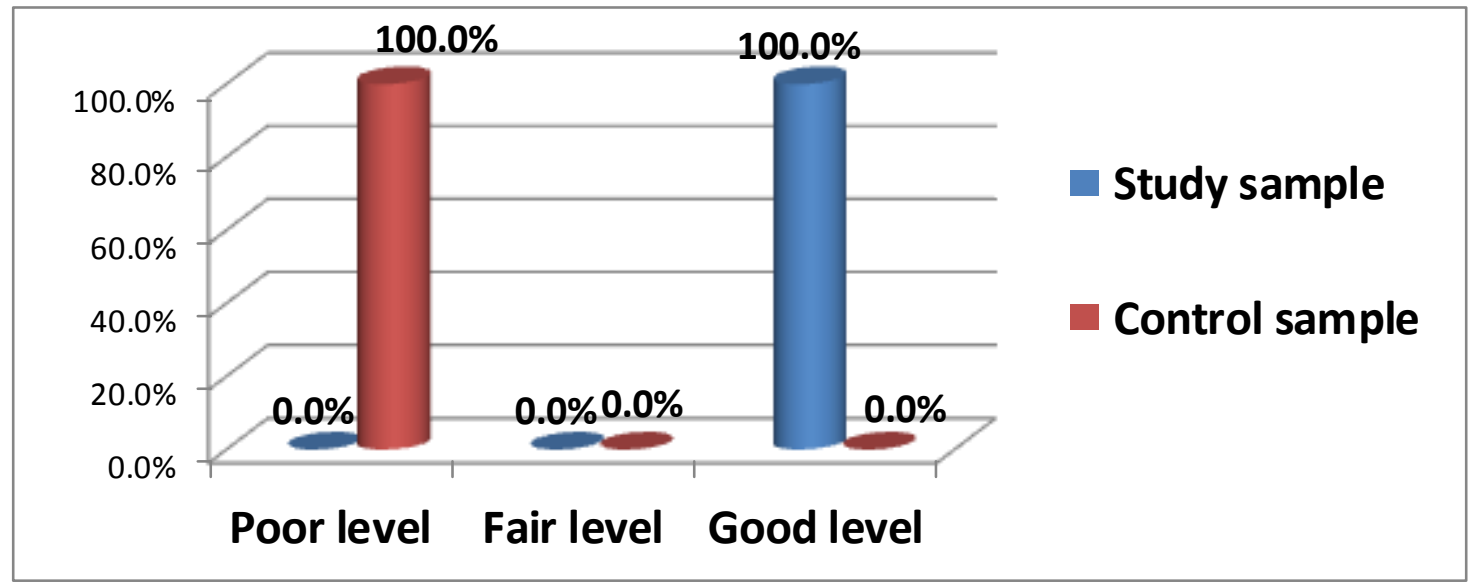

Fig. (2): Patients' Knowledge Post-Test About Coronary Artery Stent.

Table (3): Comparison between the control and study samples for immediately post operation complications.

\begin{tabular}{|c|c|c|c|c|c|c|c|c|c|}
\hline \multirow{4}{*}{ Items } & \multicolumn{8}{|c|}{ Post operation complications } & \multirow{4}{*}{$P$ value } \\
\hline & \multicolumn{4}{|c|}{ Study sample } & \multicolumn{4}{|c|}{ Control sample } & \\
\hline & \multicolumn{2}{|c|}{ Present } & \multicolumn{2}{|c|}{ Not present } & \multicolumn{2}{|c|}{ Present } & \multicolumn{2}{|c|}{ Not present } & \\
\hline & N. & $\%$ & $\mathbf{N}$. & $\%$ & $\mathbf{N}$. & $\%$ & N. & $\%$ & \\
\hline \multicolumn{10}{|l|}{ I-Immediate complications } \\
\hline \multicolumn{10}{|l|}{ 1- Local complications } \\
\hline A- Infection at the catheter insertion site. & 0 & 0.0 & 30 & 100.0 & 0 & 0.0 & 30 & 100.0 & - \\
\hline B- Allergic reaction to the dye & 0 & 0.0 & 30 & 100.0 & 0 & 0.0 & 30 & 100.0 & - \\
\hline \multicolumn{10}{|l|}{ 2- Systemic complications } \\
\hline A- Circulatory complications & 1 & 3.3 & 29 & 96.7 & 1 & 3.3 & 29 & 96.7 & 1.000 \\
\hline B- Urinary complications & 0 & 0.0 & 30 & 100.0 & 0 & 0.0 & 30 & 100.0 & - \\
\hline C- Gastrointestinal complications & 3 & 10.0 & 27 & 90.0 & 4 & 13.3 & 26 & 86.7 & 0.543 \\
\hline II- Late complications & 1 & 3.3 & 29 & 96.7 & 0 & 0.0 & 30 & 100.0 & $0.086^{*}$ \\
\hline
\end{tabular}

Independent sample T-test $*$ Statistical significant differences $(p \leq 0.05)$

Table (4): Comparison between the control and study samples for after 3 months operation complications.

\begin{tabular}{|c|c|c|c|c|c|c|c|c|c|}
\hline \multirow{4}{*}{ Items } & \multicolumn{8}{|c|}{ After 3 months operation complications } & \multirow{4}{*}{$P$ value } \\
\hline & \multicolumn{4}{|c|}{ Study sample } & \multicolumn{4}{|c|}{ Control sample } & \\
\hline & \multicolumn{2}{|c|}{ Present } & \multicolumn{2}{|c|}{ Not present } & \multicolumn{2}{|c|}{ Present } & \multicolumn{2}{|c|}{ Not present } & \\
\hline & $\mathbf{N}$. & $\%$ & N. & $\%$ & $\mathbf{N}$. & $\%$ & $\mathbf{N}$. & $\%$ & \\
\hline \multicolumn{10}{|l|}{ I-Immediate complications } \\
\hline \multicolumn{10}{|l|}{ 1- Local complications } \\
\hline $\begin{array}{l}\text { A- Infection at the catheter } \\
\text { insertion site. }\end{array}$ & 0 & 0.0 & 30 & 100.0 & 0 & 0.0 & 30 & 100.0 & - \\
\hline B- Allergic reaction to the dye & 0 & 0.0 & 30 & 100.0 & 0 & 0.0 & 30 & 100.0 & - \\
\hline \multicolumn{10}{|l|}{ 2- Systemic complications } \\
\hline A- Circulatory complications & 0 & 0.0 & 30 & 100.0 & 0 & 0.0 & 30 & 100.0 & - \\
\hline B- Urinary complications & 0 & 0.0 & 30 & 100.0 & 0 & 0.0 & 30 & 100.0 & - \\
\hline C- Gastrointestinal complications & 0 & 0.0 & 30 & 100.0 & 0 & 0.0 & 30 & 100.0 & - \\
\hline II- Late complications & 1 & 3.3 & 29 & 96.7 & 2 & 6.7 & 28 & 93.3 & $0.023^{*}$ \\
\hline
\end{tabular}

Independent sample T-test* Statistical significant differences $(p \leq 0.05$ 
Table (1): shows that most of the patients are male in both samples $(76.7 \%, 66.7 \%$ respectively). More than half of patients their age ranged from $41<60$ years in both samples $(66.7 \%, 70.0 \%$ respectively).The vast majority of patients are married (93.3\%, $96.7 \%$ respectively). More than half of patients are illiterate $(56.7 \%, 63.3 \%$ respectively). Nearly half of patients are employees $(43.3 \%, 26.7 \%$ respectively).

Table (2): found that vast majority of the patients in both samples within the normal physical examination.But as psychologically most of the patients in the control sample $(80.0 \%)$ complained of fear, also all of the patients in the study sample $(100.0 \%)$, and the vast majority of them in the control sample(93.3\%) complained of anxiety.

Fig. $(1,2)$ : shows that $100 \%, 100 \%$ of both the study and the control samples having poor knowledge score in the pre-test. While in post-test $100 \%$ of the study samples were having the good level knowledge, and the control samples still having a poor level in knowledge post-test.

Table $(3,4)$ : illustrated that no significant statistical differences between control and study samples in relation to local and circulatory complications immediately post procedure and after 3 months

\section{Discussion}

This study aimed to investigate the impact of the designed nursing educational protocol on health promotion for patients undergoing coronary artery stent outcome.

The discussion was covered the main findings as the following

- Demographic characteristics of patients

Based on the results of the present study; the majority of both groups in PCI with coronary stent were in age

group 50 to 60 years old and most patients were males.This can be attributed to the higher exposure to life

stress, and female hormones protect female from CAD. This in line with Andrea et al., (2010) who studied early sheath removal and ambulation in patients submitted to PCI: A randomized clinical trial, found that $64 \%$ of the study sample was males, and their mean age were $59.7 \%$ years old.

The current study revealed that the majority of the studied patients were married. This is in line with Basuny, (2009), study about the effect of position changing post coronary angiography on patient's outcomes, which revealed that the majority of the studied sample was married. This may attribute to the high level of daily life stress on married patients than a single one and that stress is considered one of the most aggravating factors for CAD. Both moderate and intense physical and mental activities were associated with ischemic episodes. Some studies estimated that in the hour after high levels of negative emotions, the risk for ischemia double and this is because the effects of mental stress are that stenosed coronary artery segments responded to mental stress by constricting, whereas normal segment typically responded by dilating. Colombo \& Merlini, (2009) The present study shows that $36.7,30.0$ of the study sample and 30.0, 53.3 of the control sample of patients undergoing PCI with coronary stent had hypertension and diabetes mellitus. Abdel Ghany, 2009 agree with the current study reported that $36.7 \%$ of the study had hypertension and $49 \%$ had diabetes mellitus. Also, Lunde'n et al., (2006) stated that cardiac catheterization is more common in people who suffer from medical diagnosis (ischemic heart disease) and coronary heart disease associated with hypertension, diabetes mellitus, and renal diseases.

- Patients knowledge about coronary artery stent The current study shows the majority of the patient had no information and knowledge about cardiac catheterization and the medical and nursing staff did not provide them this led to anxiety and fear from procedure.Many studied state that the effect of giving information to patients on their fear and satisfaction level as Reed et al., (2008), findings of reducing patient anxiety pre-cardiac catheterization through education.

Also, Elsaid, (2010), found that the patients education material is either sensory or procedural oriented. Procedural material describes the steps involved in the cardiac catheterization. Sensory information focuses on the patient will see and hear during the procedure helps the patients reduce discomfort.

The current study reflected that there is significant improvement of all parameters in response to the given information among the study sample in comparison with the control sample that still have poor knowledge score, this is in line with Mahgoub, (2013) study about Impact of a Designed Early Ambulation Protocol on Patients' Outcome post Transfemoral Coronary Procedures which revealed that The post mean knowledge scores of patients who will be exposed to a designed early ambulation protocol will be higher than their pre mean knowledge scores among PCI and CA as compared to a control group

- Assessment of patient complications after the procedure

The current study shows that no statistically significant differences between both groups for patients undergoing to PCI in relation to bleeding, hematoma. Kalapatapu et al., (2006), indicated that some estimate that complications related to the access 
site result in more than 75,000 surgical procedures annually. Muller et al., (2007), performed that a goal following cardiac catheterization is to reduce vascular complications, especially hematoma formation, the most common access site complication.

However, Agostoni et al., (2006), stated that the incidence of vascular access complications alone has ranged from $0.1 \%$ to $61 \%$, depending on the definition of complications, the type of procedure, anticoagulation, closure devices, age, sex, and other patient co-morbidities. Dumont et al., (2006), added vascular complications expose patients to additional discomfort, extended hospital stay, and higher hospital costs.

Also Nathan, (2013), reported that the incidence of Perioperative complications with non-cardiac surgery post second generation Drug Eluting Stent (DES) implantation was $11 \%$ and consisted mainly of bleeding $(5.8 \%)$. The incidence of definite stent thrombosis was $0.7 \%$

\section{Conclusions}

Based on the results of the present study, it can be concluded that

- Patient's knowledge regarding coronary artery stent and procedure of PCI in catheterization lab at Assiut University Hospital in protest are inadequate.

- Application of teaching protocol when dealing with coronary artery stent patients shows a significant improvement in Patient's knowledge.

\section{Recommendations}

Based on the results of the present study it can be Recommended

On the educational level

- Equip the cardiac catheterization unit with simply illustrated guidelines protocol covering PCI Procedure and coronary stent practices knowledge pre/post cardiac catheterization

- Develop an in service audiovisual materials training/education about the coronary stent, types, indications, complications and the instructions before, during and after the procedure for patients.

On the administration level

- Establishing a standardized care protocol for coronary artery stent before, during and after PCI procedure.

- Developing strategies aiming at improving the quality of care before, during and after PCI procedure.

On the practical level

- Nurses should be encouraged to attend specific meetings as workshops and seminars held for cardiac diseases and cardiac surgery to be acquainted with the most advanced and skills in this area.

- Insure training program of specialized cardiac care of patients before, during and after PCI procedure.

On the research level

- Reapply this research on a larger probability sample acquired from different geographical areas in Egypt for generalization.

- Studying the risk factors associated with local complications for patient undergoing cardiac catheterization.

- Studying to evaluate the effect of using other vascular closure devices after cardiac catheterization on patient's outcome.

- Assessment of nurse's knowledge and practices in relation to a bundle of care provided for patients undergoing coronary artery stent.

\section{References}

1. Abdel-Ghany A., (2009): Therapeutic value of PTCA in early stages of acute MI. Doctoral thesis, Faculty of Medicine, Assiut University. P 133.

2. Agostoni P., Anselmi M., \& Gasparini G., (2006): Safety of percutaneous left heart catheterization directly performed by cardiology fellows: a cohort analysis. J Invasive Cardio Jun;18(6): 248-52.

3. American Heart Association, (2010): (Cardiac procedures and surgeries, published on the internet from://www.heart.org

4. Andrea A., Quadros A., Sarmento \& Leite R., (2010): Early sheath removal and ambulation in patients submitted to percutaneous coronary intervention: A randomized clinical trial International Journal of Nursing Studies 47 939945. Published on the internet from www.elsevier.com/ijnsbased? Images 2003 Fall; 22(3):9-12.

5. Basuny S., (2009): The effect of positioning changing post coronary angiography on patient's outcomes, Master thesis, Faculty of Nursing, Alexandria University. P 144

6. British Heart Foundation, (2008): Managing patients with coronary stents; published on the internet from www.bhf.org.uk.

7. Colombo A., \& Merlini P., (2009): The ischemia/bleeding balance in PCI. Published on the internet from www.patient.co.uk/doctor/percutaneouscoronary-intervention.

8. Dumont C., Keeling A., Bourguignon C., Sarembock I., \&Turner M., (2006): Predictors of vascular complications post diagnostic cardiac 
catheterization and percutaneous interventions. Dimens Crit Care Nurs.;25(3):137-142

9. El said H., (2010): Developing nursing care guidelines for patients undergoing to cardiac catheterization based on their needs. Master Thesis, Faculty of Nursing, Alexandria University

10. European Society of Cardiology (2010): Guidelines for Myocardial Revascularisation, Available at http://www.escardio.org

11. George A., \& Josh W., (2010): Percutaneous Coronary Intervention, http://emedicine.medscape.com/article/.

12. Kalapatapu V., Ali A., \& Masroor F., (2006): Techniques for managing complications of arterial closure devices. Vasc Endovascular Surge. Oct-Nov;40(5): 399-408.

13. Lewis L., Heitkemper M., Dirksen R., Obrien G., \& Linda B., (2007): Medical Surgical Nursing., 7th edition, Elsevier, New Delhi, p. 1531.

14. Lisa T., Robert S., Gerancher J., Michael A., \& Roger L., (2010): Coronary Artery Stents: Preoperative Considerations and Management.www.ansthesia-anlgeisia.org

15. Lunde' n, M., Bengtson, A., \& Lundgren, S., (2006): Hours during and after coronary intervention and angiography. Clinical Nursing Research 15 (Nov. 4), 274-289

16. Mahgoub A., (2013): Impact of a Designed Early Ambulation Protocol on Patients' Outcome post-Transfemoral Coronary Procedures, Doctoral thesis, Faculty of Nursing, Assiut University. P 66.

17. Morton G.,\& Fontaine K., (2009): Critical Care Nursing, Lippincott Williams and Wilkins, Tokyo, p. 200-230.

18. Muller D., Shamir K., Ellis S., \& Topol E., (2007): Peripheral vascular complications after conventional and complex percutaneous coronary interventional procedures. Am J Cardiol;69:63-68

19. Nathan N., (2013): The incidence of preoperative complications with non-cardiac surgery. Available http://www.sciencdirect.com.

20. Osborn S., Wraa E., \& Watson A., (2010): Medical Surgical Nursing, Person education Inc., USA, p.p. 1106-1115.

21. Reed F., Blake G., \& Ohno-Machado L., (2008): Vascular closure devices and the risk of vascular complications after Percutaneous coronary intervention in patients receiving glycoprotein IIb-IIIa inhibitors. Am J Cardiol;88:493-496
22. Roger W., Kenneth G., Philip T., \&Scott C., \& Sheldon G., (2010): Angioplasty. National Heart, Journal report

23. Sujata K., (2010): Biomaterials for clinical applications, ch 2 coronary artery disease, Springer Science+ Business media, USA, P. 33

24. Urden L., Stacy K., \& cough M., (2006): Thelan's critical care nursing, Diagnosis, and management, ch. 15, Cardiovascular anatomy and physiology 15th ed., Mosby Inc. P 280,281 